\title{
A Size-Matching Strategy to Differentiate Flexible Conformers for the Discovery of Novel Cages with Twin Cavities
}

Zhenyu Yang, Chunyang Yu, Lihua Chen, Pan Li, Jiaolong Chen, Kim Jiayi Wu, Qiangyu Zhu, Yuquan Zhao Xiaoning Liu, and Shaodong Zhang*

Abstract: Considerable efforts have been made to characterize the meaningful conformers that a molecule can adopt, which is of great significance for understanding the structure-property correlation in the fileds of molecular biology, drug discovery, catalysis, materials science, etc. It is however challenging to differentiate and make use of the flexible conformers in solution, as they often experience rapid interconversion due to low isomerization barrier. We herein present a novel yet simple size-matching strategy for conformation identification. As a proof of concept, we rationally designed a three-dimensional model compound, namely hexaformyl molecule 1 exhibiting two types of conformers, i.e. Conformer-1 and $\mathbf{- 2}$ with different cleft positions and sizes. Aided by DFT calculations, we selected two triamino conformation capturers (denoted CC). Small-sized CC-1 selectively captured Conformer-1 by matching its cleft size, while large-sized CC-2 was able to match and capture both conformers. It therefore allowed facile differentiation of the two conformations by conventional NMR and X-ray analyses. These two erstwhile inverconverting and indistinguishable conformational isomers were made use of, leading to the discovery of two novel configurational isomers, namely two novel cage-like compounds with twin cavities, one exhibiting a sandglass-shaped and another with a dumbbell form, which we coined diphane. 


\section{INTRODUCTION}

Conformational isomers, or conformers, are interconvertible stereoisomers through free rotation around the covalent single $\sigma$ bonds and/or $s p^{3}$-hybridized centers of a molecule. ${ }^{1}$ Identifying the meaningful conformers is of great importance for elaborating the structure-property correlation in the fields of molecular biology, ${ }^{2}$ drug discovery, ${ }^{3}$ catalysis, ${ }^{4}$ materials science, ${ }^{5}$ and so on. On the other hand, by virtue of their low isomerization barrier, flexible conformers often experience spontaneous interconversion in solution, and their identification poses a formidable challenge for conventional methods with low timescale resolution, such as nuclear magnetic resonance (NMR) and infrared (IR) spectroscopies. X-ray crystallography is a powerful method to unambiguously determine the absolute conformation of a molecule, but it often only distinguishes the conformer that provides the most efficient molecular packing. ${ }^{6}$ This identification challenge also hurdles the presice handling and transformation of specific conformational isomers for the discovering of novel chemicals and/or functions.

Alternative strategies therefore have been developed, which in principle are to attenuate or quench the conformational exchange. For example, the rate of conformational isomerization can be reduced by decreasing the operational temperature, so that various conformers might become distinguishable by variable-temperature $\mathrm{NMR}^{7}$ and time-resolved spectroscopies. ${ }^{8}$ As pioneered by Fujita et al., ${ }^{9}$ once the erstwhile disordered and non-crystalline molecules are absorbed within a porous crystalline sponge, their conformations are confined, allowing direct structural determination by X-ray analysis. ${ }^{10}$ When the conformers are tethered to the electrodes, their structures are rigidified, which can also be resolved by combining single-molecule techniques and theoretical calculation. ${ }^{11}$ Inspired by this principle of conformation rigidification, we wondered if it can be applied to capture the conformational precusors by directly reacting them with other molecular entities (denoted molecular capturers) in solution. It would allow easy structural inspection by conventional characterization methods, and also leads to the discovery of novel structures that are previsously inaccessible; to our surprise, however, such simple method had remained unexplored. 


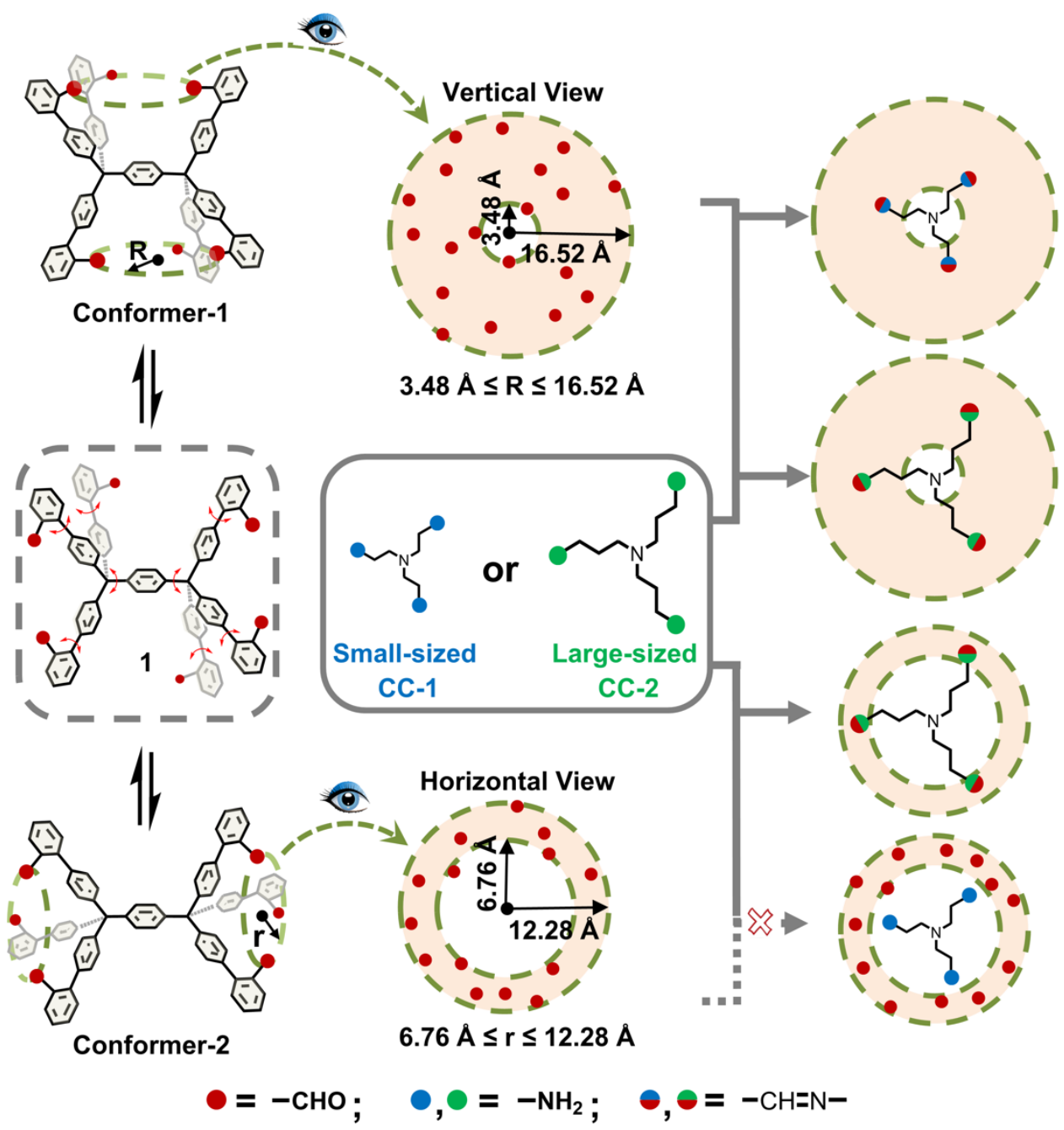

Figure 1. Schematic representation of size-matching strategy for facile identification of two types of conformations of model compound $\mathbf{1}$ with six formyl groups presented with red dots. The three-dimensional molecule $\mathbf{1}$ experiences spontaneous interconversion between Conformer-1 with a pair of upper and lower clefts and Conformer-2 with a pair of left and right clefts (left column). As revealed by DFT calculation (B3LYP/6-31G*), the permanently moving formyl groups are spotted within a projected circular ring (middle column), which is used to quantify the size of the clefts. Considering the inner circle of the ring, i.e., the smallest possible size of the cleft, is different for Conformer$\mathbf{1}$ and -2, a small-sized CC-1 (with the amines presented with blue dots) and large-sized CC-2 (with the amines presented with blue dots) were selected as size-matching conformational capturers by DFT. Small-sized CC-1 exclusively captures and identifies Conformer-1, and large-sized CC-2 captures both Conformer-1 and -2 (right column).

Our group have been focusing on the elaboration ${ }^{12}$ and hierarchical self-assembly ${ }^{13}$ of covalent organic cages prepared by dynamic covalent chemistry (DCC). ${ }^{14}$ Due to the dynamic and reversible nature of DCC, it allows error correction to yield the thermodynamic products. ${ }^{15}$ This feature prompted us to rationally design a three dimensional (3D) model compound 1 with density-functional theory (DFT) calculation (left column, Figure 1). This hexaformyl molecule 1 exhibits all possible conformations that fall into two distinct types: Conformer-1 with two clefts located below and above the central arene, in each cleft three formyl groups tending to point inward; Conformer-2 with a pair of left and right clefts, where each three formyl 
moieties are outward positioned. Due to the molecular motion, the size of these clefts varies spontaneously, with the permanently moving formyl groups forming a circular ring (middle column, Figure 1). As the size range of the clefts of Conformer-1 and -2 are different, we developed a size-matching strategy by choosing two triamino conformation capturers (abbreviated CC), i.e., CC-1 and -2 with different sizes. Through defect-checking DCC, these two molecular capturers can chemically capture Conformer-1 and $\mathbf{- 2}$ (right column, Figure 1), which allowed their facile differentiation by conventional NMR and X-ray analyses, and also led to the formation of two novel cage-like compounds with twin cavities (Figure 3), ${ }^{16}$ which we coined diphane.

\section{RESULTS AND DISCUSSION}

Design and characterization of model compound. As briefly mentioned above, the geometry optimizatoins (DFT, B3LYP/6-31G*) showed that molecule 1 exists infinite forms of conformers via all possible $\mathrm{C}-\mathrm{C}$ single bond rotations, from which two types of conformers, Conformer-1 and -2, were identified (Figure 1 and Figure 2a). It revealed that Conformer-1 exhibits two clefts located below and above the central arene, in each of which three formyl groups tend to point inward (left, Figure 2a); while in Conformer-2 each three formyl moieties are outward positioned (right, Figure 2a). The energy difference of the two conformers is extremely low $\left(c a .1 .1 \mathrm{~kJ} \mathrm{~mol}^{-1}\right)$, indicating their fast interconversion, which could have been otherwise challenging to be distinguished by conventional analytical tools. Accordingly, molecule 1 was sythesized by $\mathrm{Pd}\left(\mathrm{PPh}_{3}\right)_{4}$ catalyzed Suzuki-Miyaura cross-coupling reaction using commercially available molecule (2-formyl phenyl)boronic acid as reactant (51\% five-step overall yield, see Supporting Information for details). The rapid averaging of its possible conformers was confirmed by ${ }^{1} \mathrm{H}$ NMR spectroscopy at $298 \mathrm{~K}$, with symmetric splitting patterns of all protons (Figure 2b). Its single crystals suitable for X-ray crystallography were obtained by slow evaporation of solvent from its chloroform solution, but only Conformer-1 could be identified (Figure 2c). 
(a)

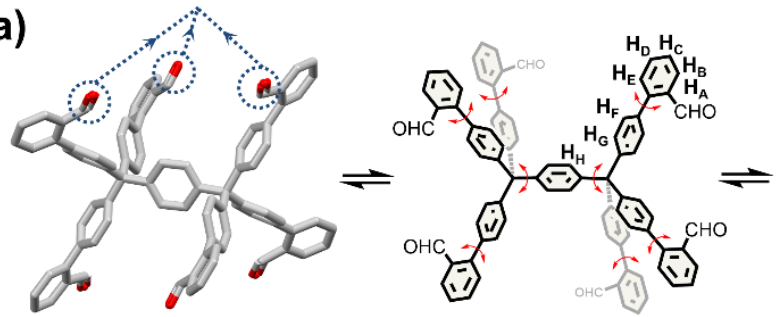

Conformer-1

$\left(0 \mathrm{~kJ} \mathrm{~mol}^{-1}\right)$

(b)

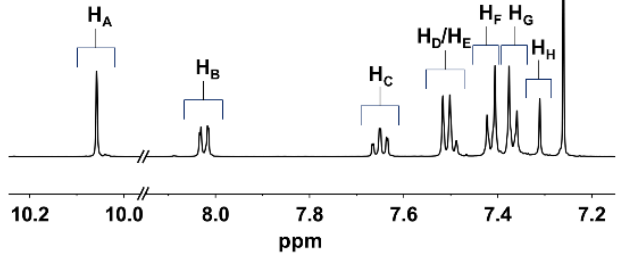

(c)

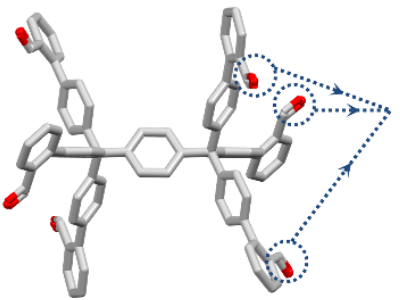

Conformer-2

$\left(-1.13 \mathrm{~kJ} \mathrm{~mol}^{-1}\right)$

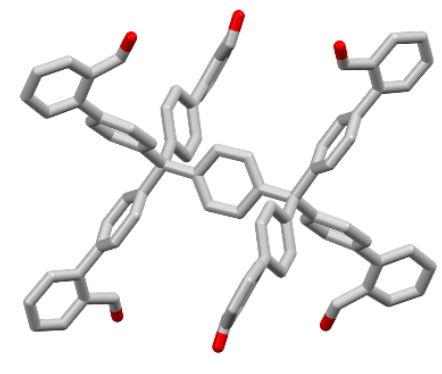

Conformer-1

Figure 2. Conformational interconversion of molecule $\mathbf{1}$ and its identification by NMR spectroscopy and SC-XRD. (a) Fast interconversion of Conformer-1 and -2 with extremely low isomerization barrier. Free energy of each isomer was determined by geometry optimization (DFT, B3LYP/6-31G*). (b) Partial ${ }^{1} \mathrm{H}$ NMR spectrum (400 MHz, $\mathrm{CDCl}_{3}$, 298K) of molecule 1 in $\mathrm{CDCl}_{3}$, showing rapid averaging of its possible conformers. (c) Molecular structure of molecule 1 revealed by SC-XRD, only able to identify Conformer-1 in solid state. Solvent molecules and hydrogen atoms are omitted for clarity. Carbon atoms are labeled in gray, and oxygen in red. Dashed line are used to facilitate the inspection of the orientation of formyl groups.

Conformation capturing with diphane formation. Covalent organic cages have attracted increasing interest in the recent decade, ${ }^{14}$ and their porosity and geometric diversity have widely been explored for selective recognition/separation, ${ }^{17}$ catalysis, ${ }^{18}$ hierarchical self-asssembly, ${ }^{13}$ etc. As the framework of cages can experience complex motions in space-including continuous bending, twisting, stretching, and shrinking, they also provide an excellent platform for the study of molecular conformation and topology. By taking advantage of the dynamic and reversible nature of DCC, and facilitated by DFT calculation (vide infra), we chose CC-1 and -2 with flexible amine-capped chains and different sizes to tentatively capture the above-mentioned conformations of molecule 1 by error-checking imine formation (Figure 3, and Figure S5 and S6 in the Supporting Information).

We first used the small-size CC-1, i.e., tris(2-aminoethyl)amine, to react with molecule $\mathbf{1}$ in the presence of $\mathrm{Sc}(\mathrm{OTf})_{3}$ with a molar ratio of $\mathbf{C C - 1 : 1 : ~} \mathrm{Sc}(\mathrm{OTf})_{3}=2: 1: 0.6$ in chloroform, and the reaction mixture was then subjected to reduction with $\mathrm{NaHB}(\mathrm{OAc})_{3}$ (15 equiv.), yielding the amine-containing product for easy purification (64\% two-step overall yield, see Supporting Information for details). The resulting product was examined by matrix-assisted laser desorption/ionization time-of-flight mass 
spectroscopy (MALDI-TOF MS), ${ }^{1} \mathrm{H}$ and COSY NMR and single crystal X-ray diffraction (SC-XRD). MALDI-TOF MS showed a single ion peak at $\mathrm{m} / \mathrm{z} 1384.7883$, corresponding to the expected product with the formula $\mathrm{C}_{98} \mathrm{H}_{94} \mathrm{~N}_{8}\left([\mathrm{M}+\mathrm{H}]^{+}\right.$calcd for 1384.7708$)$.

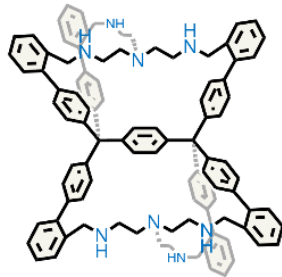

endo-[1,2,4]diphane

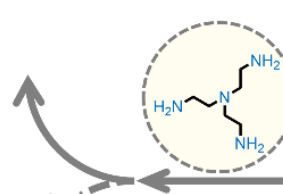

is Small-sized: CC-1

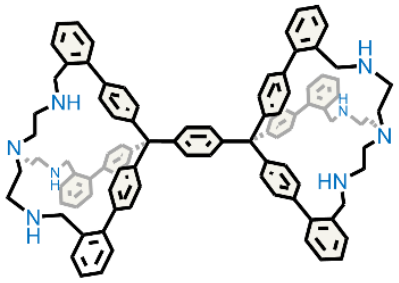

exo-[1,2,4]diphane
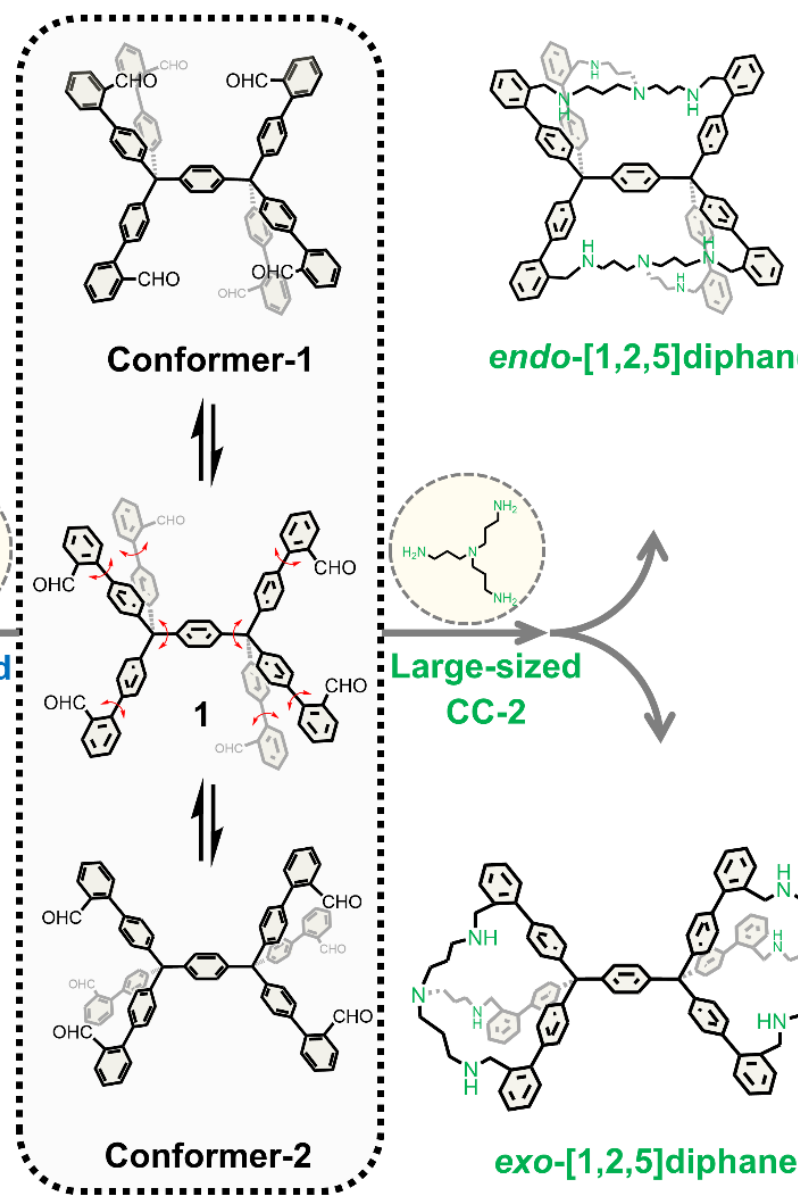

endo-[1,2,5]diphane
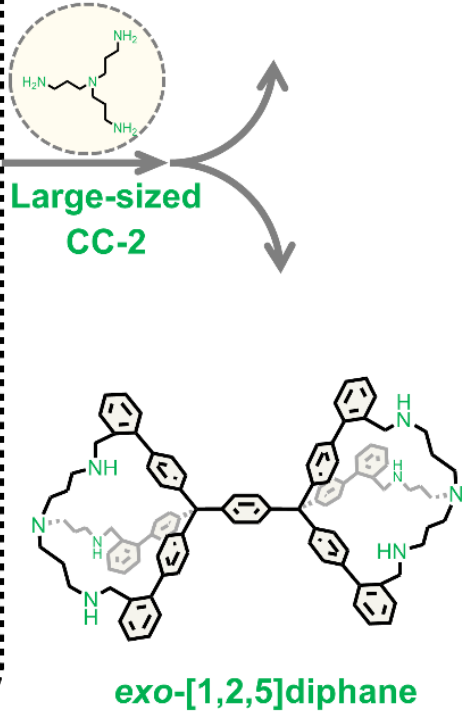

Figure 3. Schematic representation of conformation capturing of two representative and meaningful conformers, i.e., Conformer-1 and -2 of model compound $\mathbf{1}$ among its infinite interconvertible conformers in solution, achieved by dynamic and reversible cycloimination and subsequent reduction. Small-sized conformer capturer $\mathbf{C C}-\mathbf{1}$ exclusively captured Conformer-1, leading to the formation of endo-[1,2,4]diphane. Large-sized CC-2 captured both Conformer-1 and -2, yielding endo-[1,2,5]diphane and exo-[1,2,5]diphane, respectively.

The single crystals suitable for X-ray analysis were obtained by slow evaporation of its THF solution with additional trifluoroacetic acid (TFA) for better solubility. It crystallized into triclinic space group $P \overline{1}$, and unambigueously revealed only one isomer corresponding to Conformer-1 (Figure 4a), in which the central arene is shared by two identical cavities. As this twin-cavity cage-like compound is composed of aromatic units and aliphatic chains, we therefore name it diphane. This molecule is given a systematic name endo-[1,2,4]diphane, where "endo" indicates the central arene is embedded within the twin cavities, "1" refers to the number of central benzene rings, " 2 " denotes the number of aromatic units of every rigid arm, and "4" counts the backbone atoms forming each flexible chain. Due to the significant intramolecular strain, 
the aliphatic chains are twisted in an irregular fashion. The twisting of these three chains are highly uneven (Figure $4 \mathrm{~b}$ ), in agreement with the complex yet distinct splitting of each proton in ${ }^{1} \mathrm{H}$ NMR spectrum (Figure 4c). These results therefore indicate that our tethering strategy with conformer capturers provides an efficient way to "visualize" the flexible conformers in solution, with an added bonus of discovering novel 3D structures with appealing topology.

(a)

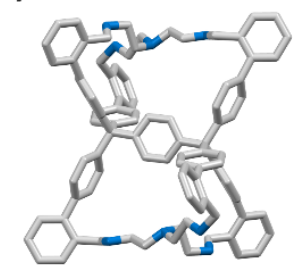

(d)

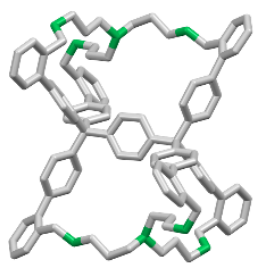

(g)

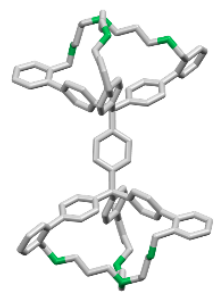

(b)

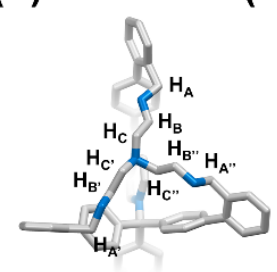

(c)

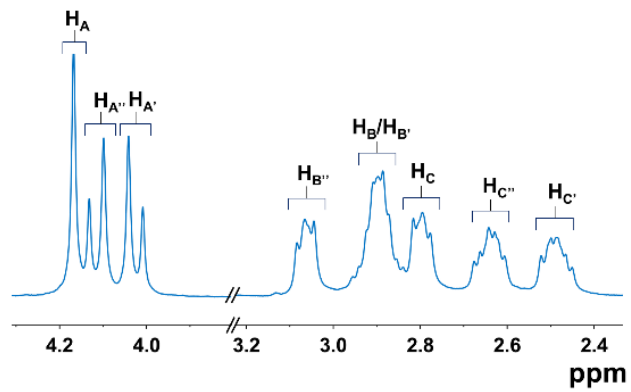

(e)

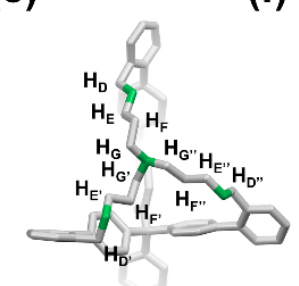

(f)

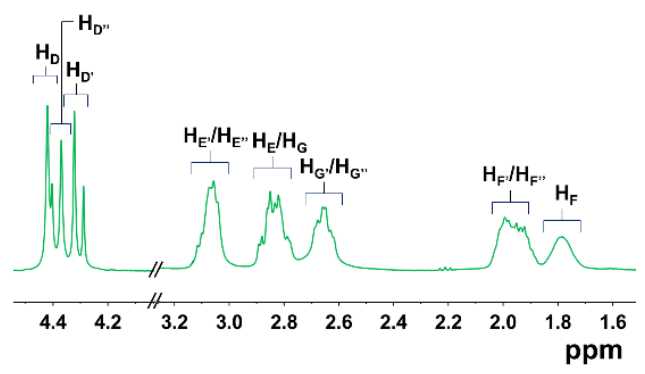

(h)

(i)

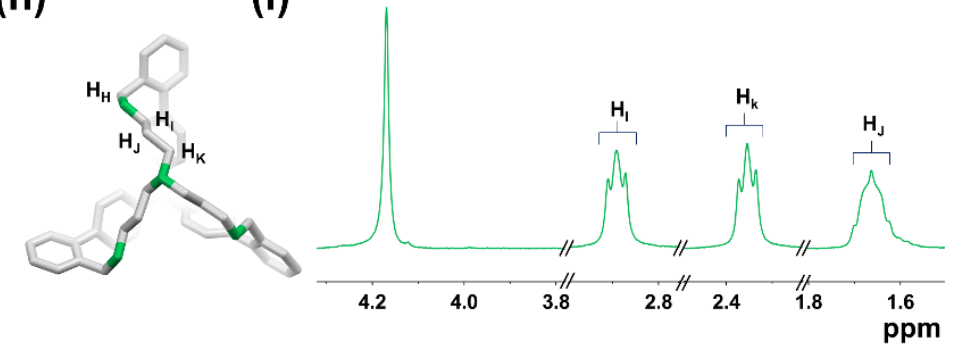

Figure 4. Moleculer structures of three diphanes determined by SC-XRD and ${ }^{1} \mathrm{H}$ NMR spectroscopy. Side views (a, $\mathrm{d}, \mathrm{g}$ ), trancated top views (b, e, h), and their corresponding partial ${ }^{1} \mathrm{H}$ NMR spectra (c, f, i) of endo-[1,2,4]diphane (in blue and gray), endo-[1,2,5]diphane (in green and gray) and exo-[1,2,5]diphane (in green and gray), respectively. Hydrogen atoms and solvent molecules are omitted for clarity. Carbon atoms are labelled in gray, and nitrogen in blue and green to distinguish the shorter and longer flexible chains, respectively.

On the other hand, we failed to capture Conformer-2 by the formation of hypothetical exo$[1,2,4]$ diphane (Figure 3), where "exo" indicates the central arene is located outside the two compositional cages. This result therefore confirms the efficacy of our size-matching strategy, which theoretically rules out the formation of exo-[1,2,4]diphane, as the maximally stretched amines of CC-1 still cannot reach the three formyl groups within each cleft of Conformer-2 (vide supra, Figure 1 and Figure S4). 
We then employed the large-sized conformational capturer CC-2, namely tris(3-aminopropyl)amine, with one more carbon unit on each chain as compared to CC-1. The stoichiometric imination between $\mathbf{C C}$ $\mathbf{2}$ and molecule $\mathbf{1}$ followed by subsequent reduction yielded two isolated products with the same ion peak at $\mathrm{m} / \mathrm{z}$ of 1468.8551 , corresponding to the expected diphane isomers with the formula $\mathrm{C}_{104} \mathrm{H}_{106} \mathrm{~N}_{8}\left([\mathrm{M}+\mathrm{H}]^{+}\right.$ calcd for 148.8647). To our delight, these two products were identified as endo-[1,2,5]diphane (59\% twostep overall yield) and exo-[1,2,5]diphane (16\% two-step overall yield) by SC-XRD and NMR spectroscopy (Figure 4d-f, and g-i, respectively, see Supporting Information for details). Endo$[1,2,5]$ Diphane adopts similar conformation with endo-[1,2,4]diphane (Figure 4d-f). On the other hand, exo-[1,2,5]diphane exhibits a dumbbell-shaped structure, with the middle arene connecting two individual cavities. This dumbbell-shaped cage had been unprecedented until the very recent and separate works of Li and Stang, ${ }^{16 \mathrm{a}}$ and Greenaway and Cooper. ${ }^{16 \mathrm{~b}}$ In contrary to its endo-isomer, exo-[1,2,5]diphane manifests a highly symmetric conformation, as each aromatic arm and flexible chain are twisted and stretched in the same manner (Figure 4h). This was also confirmed by the simple splitting of each proton in ${ }^{1} \mathrm{H}$ NMR spectrum (Figure 4i).

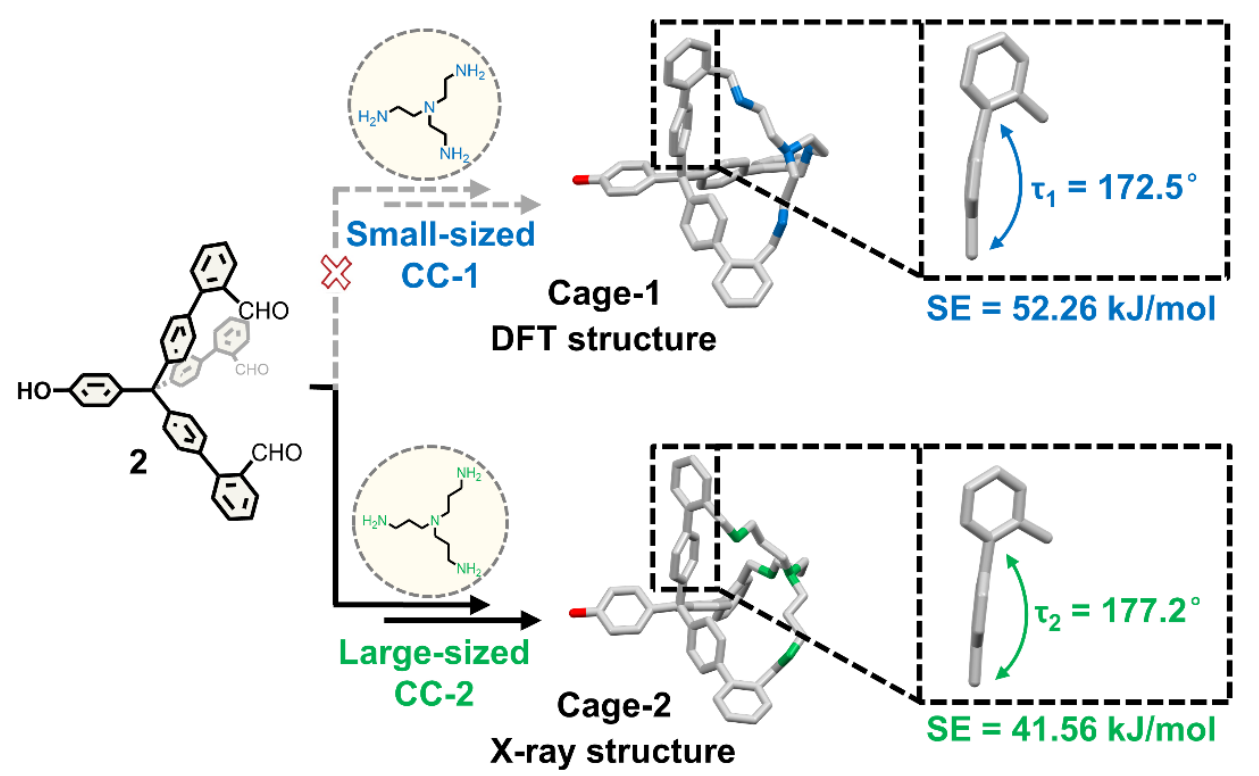

Figure 5. Inspection of size effect of conformation capturers CC-1 and CC-2 with model compound 2. Small-sized CC-1 failed to form Cage-1, which is presented here with a geometry optimized structure (DFT, B3LYP/6-31G*); While CC-2 succeeded in yielding Cage-2, with its molecular structure determined by X-ray crystallograpy. Hydrogen atoms and solvent molecules are omitted for clarity. The insets show the enlarged rigid arm of the two cages, revealing their torsion angles and the corresponding strain energies, respectively.

\section{Mechanistic study of conformation capturing and diphane formation}

In order to investigate the mechanism of exclusive capturing of Conformer-1 with small-sized CC-1 in solution, we designed the control experiments as follows (Figure 5). The triformyl model compound $\mathbf{2}$ is 
the half truncated version of molecule 1, which eliminates the formation possiblity related to the conformation of endo-[1,2,4]diphane or endo-[1,2,5]diphane. It therefore enabled the direct inspection of size effect of conformation capturers CC-1 and CC-2. When small-sized CC-1 was used, the formation of Cage-1 could only be detected by mass spectroscopy but not by NMR, which is presented here with its optimized structure by DFT (Figure 5); while Cage-2 was formed with large-sized CC-2 under the same reaction conditions (see Supporting Information for details). The single crystals suitable for X-ray analysis of Cage-2 were obtained by slowly diffusing hexane into its chloroform solution, confirming its expected structure. The strain energies (SE) of both products were also examined, and it showed that Cage-1 exhibits a much higher strain as compared to Cage-2 (52.26 vs. $41.56 \mathrm{~kJ} \mathrm{~mol}^{-1}$, Figure 5). Besides, the rigid arm of the optimized Cage-1 is significantly bent with a torsion angle of $\tau_{1}=172.5^{\circ}$, while the arm of Cage-2 is much closer to the loose conformation with $\tau_{1}=177.2^{\circ}$. This high strain of Cage-1 presumably prevents its formation, which is also true as for exo-[1,2,4]diphane (vide infra).

To gain further insight into the capturing process between Conformer-1 and -2 with the larger capturer CC-2, the kinetic experiment was carried out with ${ }^{1} \mathrm{H} \mathrm{NMR}$ at $298 \mathrm{k}$ in $\mathrm{CDCl}_{3}$ without reduction of the resulting imine bonds (Figure 6a). Initially, all possible conformers of molecule 1 interconverted rapidly in solution, and the ${ }^{1} \mathrm{H}$ NMR spectrum displayed average splitting before the addition of $\mathbf{C C}-2$ at $0 \mathrm{~h}$. Upon the addition of $\mathbf{C C - 2}$, the intensities of $\mathbf{H}_{\mathbf{A}}$ and $\mathbf{H}_{\mathbf{B}}$ of molecule $\mathbf{1}$ decreased steadily with time until their complete disapperance after four hours. Meanwhile, two groups of characteristic peaks, respectively colored in light blue and red, emerged and gradually increased until the plateau at $4 \mathrm{~h}$.

The peaks in light blue represent the formation of the imine form of endo-[1,2,5]diphane, denoted endo-[1,2,5]diphane', of which $\mathbf{H}_{\mathbf{C}}$ is the proton of shiff base and $\mathbf{H}_{\mathbf{D}}$ the aromatic proton adjacent to $\mathbf{H}_{\mathbf{C}}$. The peaks in light red are assigned to the imine form of exo-[1,2,5]diphane, namely exo-[1,2,5]diphane', of which $\mathbf{H}_{\mathbf{C}}$, corresponds to the proton of shiff base and $\mathbf{H}_{\mathbf{D}}$, the aromatic proton in the immediate vinicity of $\mathbf{H}_{\mathbf{C}}$. The evolution profile illustrates that the consumption of molecule $\mathbf{1}$ occurred simultaneously with the formation of two diphane isomers until the equilibrium. No interchange between these two imine-based isomers has been observed throughout their formation process. It means that the erstwhile fast interconversion of conformers of molecule 1 was quenched upon reacting with $\mathbf{C C - 2}$, preventing the exchange of two isomers despite the dynamic feature of DCC. We also noticed that the conversion rates of endo- and exo-[1,2,5]diphane' were different, as the former was generated faster than the latter, leading to a product distribution of $75 \%$ endo- and $25 \%$ exo-[1,2,5]diphane', respectively. 


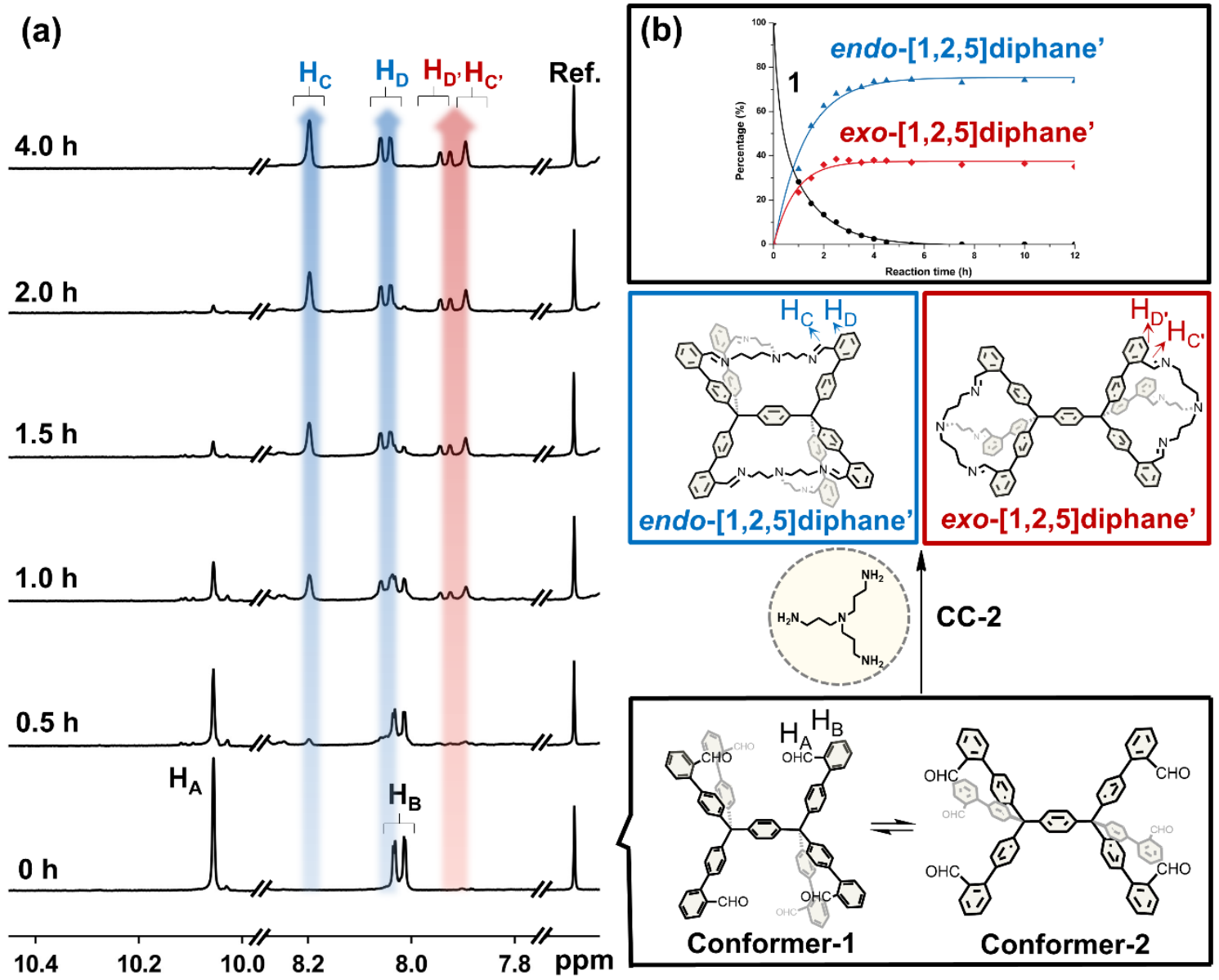

Figure 6. ${ }^{1} \mathrm{H}$ NMR study of the conformer capturing progress of Conformer-1 and $\mathbf{- 2}$ with large-sized CC-2 as a function of time. (a) The ${ }^{1} \mathrm{H}$ NMR spectra indicate that Conformer-1 and -2 were captured simutaniouly with CC-2. (b) The evolution profile of molecule 1 in gray, endo-[1,2,5]diphane' in blue and exo-[1,2,5]diphane' in red, determined by the ratios of integral areas of the corresponding characteristic peaks with the internal reference peak of dioxane. $\mathbf{H}_{\mathrm{A}}$ represents the proton of aldehyde group of molecule $\mathbf{1}, \mathbf{H}_{\mathrm{B}}$ the nearest proton to $\mathbf{H}_{\mathrm{A}} ; \mathbf{H}_{\mathrm{C}}$ the proton of schiff base of endo-[1,2,5]diphane', $\mathbf{H}_{\mathrm{D}}$ the nearest proton to $\mathbf{H}_{\mathrm{C}}$ on the rigid part of the molecule; $\mathbf{H}_{\mathbf{C}}$, and $\mathbf{H}_{\mathbf{D}}$, the corresponding protons of exo-[1,2,5]diphane'.

A parellel study was also conducted to compare the thermodynamic stability of endo-[1,2,4]diphane' formed with small-sized CC-1, and endo-[1,2,5]diphane' and exo-[1,2,5]diphane' formed with large-sized CC-2 (Figure 7). CC-1 was first added to the solution of molecule 1, forming exclusively endo$[\mathbf{1 , 2}, \mathbf{4}]$ diphane', as revealed by ${ }^{1} \mathrm{H}$ NMR $(0-3 \mathrm{~h})$. Large-sized CC-2 was then introduced to this reaction mixture. Within an additional $0.5 \mathrm{~h}$, the peaks of endo-[1,2,4]diphane' completely disappeared in the ${ }^{1} \mathrm{H}$ NMR spectrum $(3.5 \mathrm{~h})$, while the formation of endo-[1,2,5]diphane' and exo-[1,2,5]diphane' was observed. No inverconversion between these three diphanes was noticed with prolonged reaction time (3.56 h). This result clearly indicates that endo-[1,2,5]diphane' and exo-[1,2,5]diphane' are thermodynamically more stable than endo-[1,2,4]diphane'. 
(a)

(b)

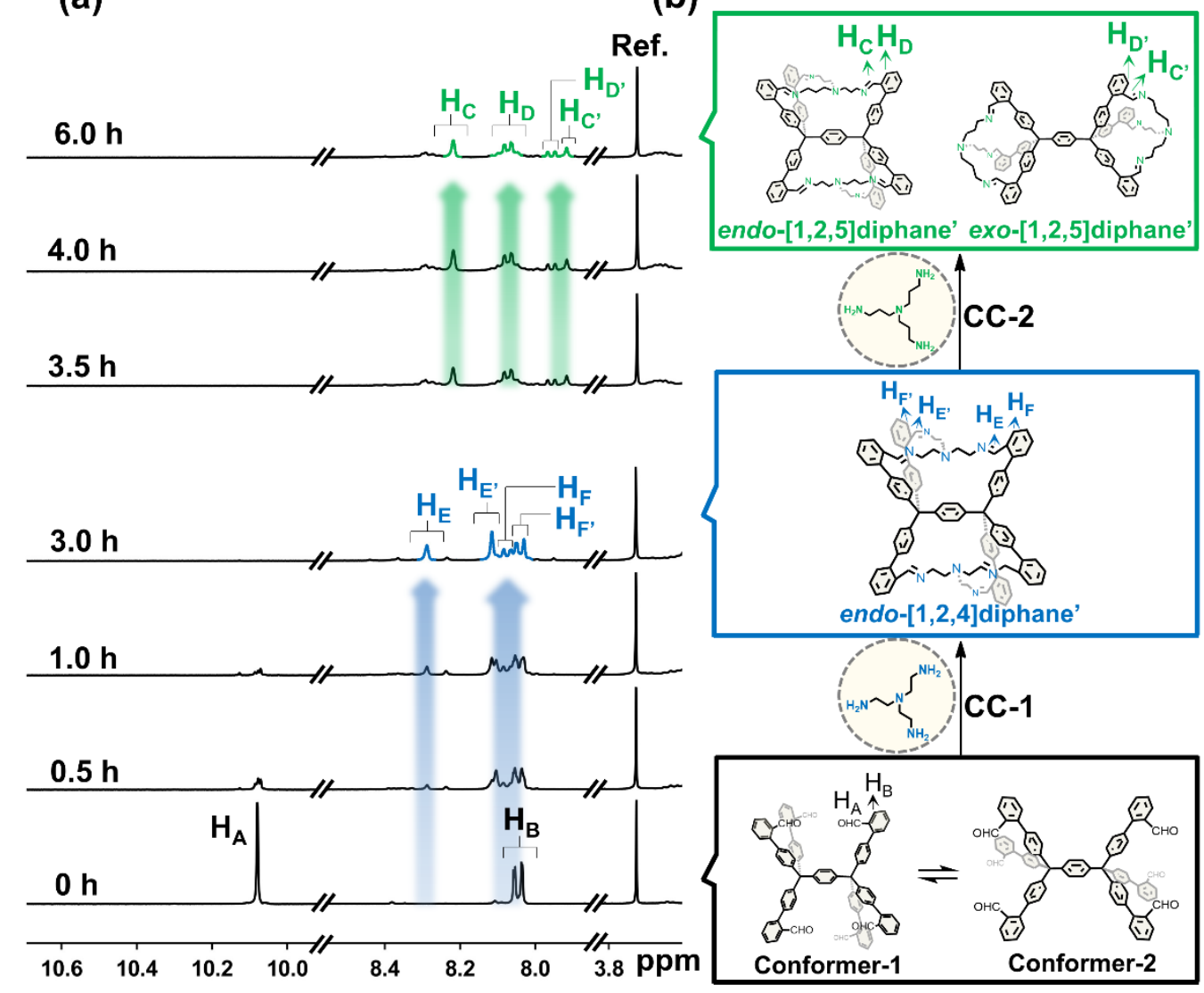

Figure 7. Transformation of endo-[1,2,4]diphane' to endo-[1,2,5]diphane' and exo-[1,2,5]diphane', followed by ${ }^{1} \mathrm{H}$ NMR study $\left(400 \mathrm{~Hz}, \mathrm{CDCl}_{3}, 2{ }^{\circ} \mathrm{C}\right.$ ). (a) The ${ }^{1} \mathrm{H}$ NMR spectra suggest that Conformer-1 was captured exclusively with CC-1 (0-3.5 h), and the resulting endo-[1,2,4]diphane' was then transformed to endo-[1,2,5]diphane' and exo$[\mathbf{1 , 2}, \mathbf{5}]$ diphane' rapidly and completely by subsequent addition of CC-2 (3.5-6 h). (b) The reaction and chemical structures with the corresponding protons are given for reference.

The difference of thermodynamic stability of all diphanes were also studied by DFT calculations (B3LYP/6-31G*), which is summarized in Figure 8. Their strain energy (SE) and torsion angle were used for such purpose. The SE of $\boldsymbol{e x \boldsymbol { o }}$-[1,2,4]diphane is the highest with $99.85 \mathrm{~kJ} \mathrm{~mol}^{-1}$, larger than $92.32 \mathrm{~kJ}$ $\mathrm{mol}^{-1}$ for endo-[1,2,4]diphane, and exo-[1,2,4]diphane is significantly bent with the smallest torsion angle of $\tau_{4}=172.5^{\circ}$, smaller than $174.0^{\circ}$ for $\boldsymbol{e n d o}-[\mathbf{1 , 2 , 4}]$ diphane. These results are in line with their truncated forms Cage-1 and Cage-2 (Figure 5). When using the large-sized CC-2, the SE values of the resulting diphanes are considerably lower, with $65.86 \mathrm{~kJ} \mathrm{~mol}^{-1}$ for endo-[1,2,5]diphane, and $77.98 \mathrm{~kJ} \mathrm{~mol}^{-1}$ for exo$[1,2,5]$ diphane, respectively, echoed with their corresponding torsion angles. The SE difference between endo-[1,2,4]diphane and exo-[1,2,4]diphane therefore presumably leads to the formation of the former as the major product (Figure 6). Considering no formation of exo-[1,2,4]diphane was observed while its SE

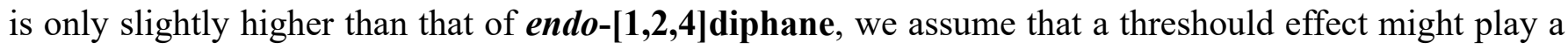
significant role that determines the formation of diphanes in the current study. 

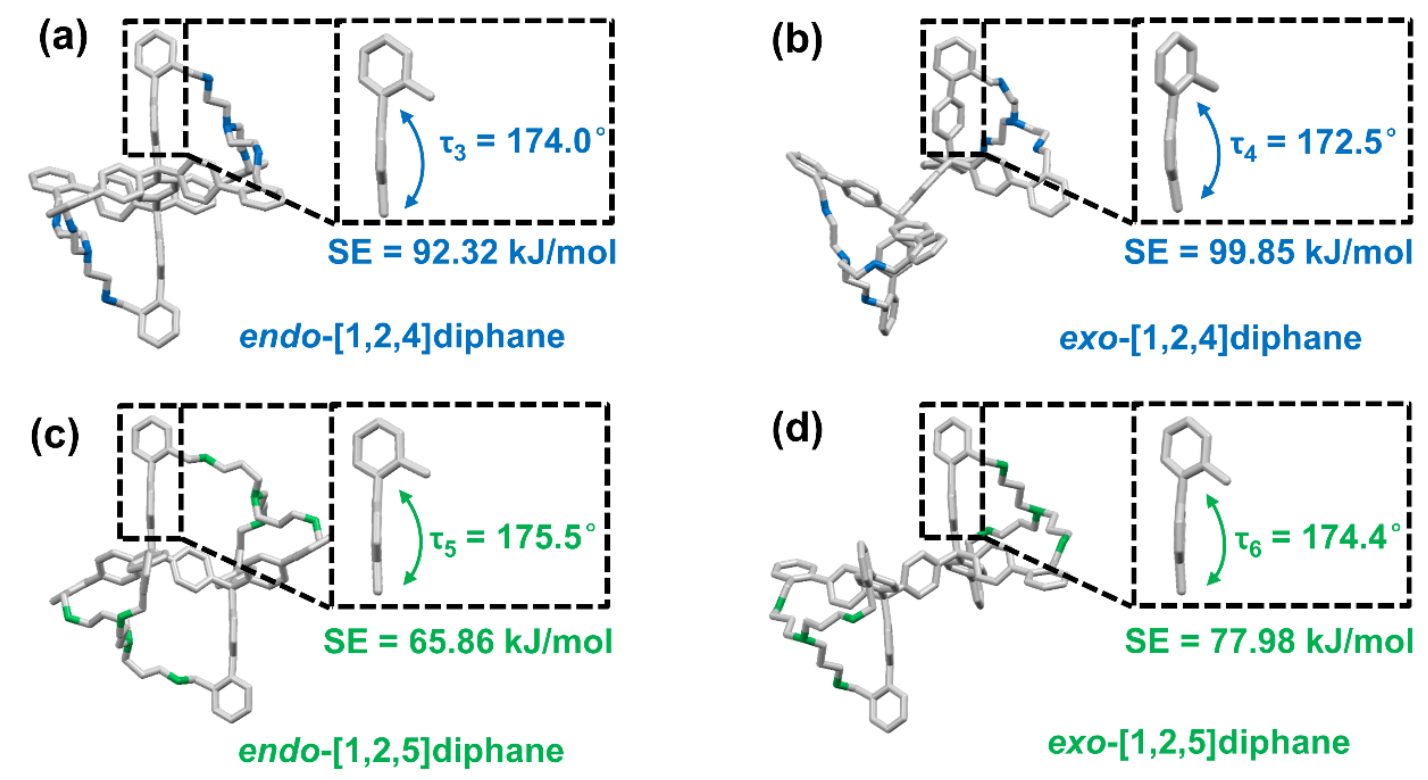

\begin{tabular}{cccc}
\hline Diphane & Strain Energy (SE) & Torsion Angle $(\tau)$ & Isolated Yield (\%) \\
\hline endo-[1,2,4]diphane & $93.32 \mathrm{~kJ} / \mathrm{mol}$ & $174.0^{\circ}$ & 64 \\
exo-[1,2,4]diphane & $99.85 \mathrm{~kJ} / \mathrm{mol}$ & $172.5^{\circ}$ & No \\
endo-[1,2,5]diphane & $65.86 \mathrm{~kJ} / \mathrm{mol}$ & $175.5^{\circ}$ & 59 \\
exo-[1,2,5]diphane & $77.98 \mathrm{~kJ} / \mathrm{mol}$ & $174.4^{\circ}$ & 16 \\
\hline
\end{tabular}

Figure 8. Summary of strain energy (SE) and torsion angles of the diphanes determined by DFT (B3LYP/6-31G*) calculations, and their corresponding isolated yields.

\section{CONCLUSIONS}

In summary, we developed a novel yet simple size-matching strategy to identify and distinguish the interconverting conformational isomers that are otherwise difficult to be distinguished by conventional analytical tools. The proof-of-concept hexaformyl model compound $\mathbf{1}$ exhibits infinite conformers that fall into two types of conformers, namely Conformer-1 with a pair of upper and lower clefts and Conformer2 with a pair of left and right clefts. As revealed by DFT calculation, the sizes of clefts are different for the two conformers. By making the most of dynamic and reversible nature of cycloimination, we chose two triamino conformation capturers, i.e., CC-1 and -2 with different sizes to match and chemically tether the two conformers with different-sized clefts. It facilitated their retrorespective differentiation by conventional NMR and X-ray analyses. This method allowed us to discover two novel cage-like compounds with twin cavities, denoted diphanes. The sandglass-shaped endo-[1,2,4]diphane and endo-[1,2,5]diphane have two 
interconnected pockets by sharing a common aromatic vertex, while the dumbbell-like exo-[1,2,4]diphane exhibits two individual apertures connected by a joint benzene ring.

This method opens up avenues for the discovery of unprecedented 3D molecules with exotic geometries and cavities. These molecules include cages with two different pockets, denoted Janus diphanes, which we will report in due course. These molecules with unique shapes and configurations can also be used novel supramolecular synthons for the construction of hierarchical superstructures ${ }^{13,19}$ with the potential application of proton conductivity, ferroelectricity, catalysis, and so on, which are the ongoing endeavors within our laboratories. 


\section{ASSOCIATED CONTENT}

\section{Supporting Information}

The Supporting Information is available free of charge.

Characterization methods, experimental details and characterization of compounds (PDF)

Crystal structures for molecule 1, Cage-2, endo-[1,2,4]diphane, endo-[1,2,5]diphane, and exo$[\mathbf{1 , 2}, \mathbf{5}]$ diphane (CIF, CIF, CIF, CIF, CIF)

\section{AUTHOR INFORMATION}

\section{Corresponding Author}

Shaodong Zhang - School of Chemistry and Chemical Engineering, Frontiers Science Centre for Transformative Molecules, Shanghai Key Laboratory of Electrical Insulation and Thermal Aging, Shanghai Jiao Tong University, 800 Dongchuan Road, Shanghai 200240, China; orcid.org/0000-0001-7923-8457; Email: sdzhang@sjtu.edu.cn.

\section{Authors}

Zhenyu Yang - School of Chemistry and Chemical Engineering, Shanghai Jiao Tong University, 800 Dongchuan Road, Shanghai 200240, China; orcid.org/0000-0001-7980-5415

Chunyang Yu - School of Chemistry and Chemical Engineering, Frontiers Science Centre for Transformative Molecules, Shanghai Key Laboratory of Electrical Insulation and Thermal Aging, Shanghai Jiao Tong University, 800 Dongchuan Road, Shanghai 200240, China; orcid.org/0000-0003-1175-8362

Lihua Chen - School of Chemistry and Chemical Engineering, Shanghai Jiao Tong University, 800 Dongchuan Road, Shanghai 200240, China; orcid.org/0000-0002-6204-9497

Pan Li - School of Chemistry and Chemical Engineering, Shanghai Jiao Tong University, 800 Dongchuan Road, Shanghai 200240, China; orcid.org/0000-0003-0901-003X

Jiaolong Chen - School of Chemistry and Chemical Engineering, Shanghai Jiao Tong University, 800 Dongchuan Road, Shanghai 200240, China; orcid.org/0000-0003-0000-9147

Kim Jiayi Wu - School of Chemistry, the University of Edinburgh, W. Mains Road, Edinburgh EH9 3FJ, United Kingdom; orcid.org/0000-0002-9726-0997

Qiangyu Zhu - School of Chemistry and Chemical Engineering, Shanghai Jiao Tong University, 800 Dongchuan Road, Shanghai 200240, China; orcid.org/0000-0002-1976-9798

Yuquan Zhao - School of Chemistry and Chemical Engineering, Shanghai Jiao Tong University, 800 Dongchuan Road, Shanghai 200240, China; orcid.org/0000-0001-7221-5471 
Xiaoning Liu - School of Chemistry and Chemical Engineering, Shanghai Jiao Tong University, 800 Dongchuan Road, Shanghai 200240, China; orcid.org/0000-0002-3725-0236

\section{Notes}

The authors declare no conflict of interest.

\section{ACKNOWLEDGMENTS}

This work was supported by National Natural Science Foundation of China (key program 21890733, and 22071153), and the Shanghai Natural Science Foundation (18ZR1420800). We thank Dr. Hang Wang (MALDI-TOF MS) and Ling-Ling Li (SC-XRD) at the Instrumental Analysis Centre of SJTU. We thank Dr. Shaohui Lin, Dr. Shan Jiang, and Dr. Liang Zhang for constructive discussion and suggestions.

\section{REFERENCES}

(1) Roos, G.; Roos, C. Isomers and Stereochemistry. In Organic Chemistry Concepts, 1st ed.; Elsevier: London, 2015; pp 43-54.

(2) (a) Möller, I. R.; Slivacka, M.; Nielsen, A. K.; Rasmussen, S. G. F.; Gether, U.; Loland, C. J.; Rand, K.

D. Conformational Dynamics of the Human Serotonin Transporter During Substrate and Drug Binding. Nat. Commun. 2019, 10, 1687; (b) Henderson, R.; Edwards, R. J.; Mansouri, K.; Janowska, K.; Stalls, V.; Gobeil, S. M. C.; Kopp, M.; Li, D.; Parks, R.; Hsu, A. L.; Borgnia, M. J.; Haynes, B. F.; Acharya, P. Controlling the SARS-CoV-2 Spike Glycoprotein Conformation. Nat. Struct. Mol. Biol. 2020, 27, 925-933.

(3) (a) McTigue, M.; Murray, B. W.; Chen, J. H.; Deng, Y.; Solowiej, J.; Kania, R. S. Molecular Conformations, Interactions, and Properties Associated with Drug Efficiency and Clinical Performance among VEGFR TK Inhibitors. Procd. Natl. Acad. Sci. U.S.A. 2012, 109, 18281-18289; (b) Over, B.; Matsson, P.; Tyrchan, C.; Artursson, P.; Doak, B. C.; Foley, M. A.; Hilgendorf, C.; Johnston, S. E.; Lee, M. D., IV; Lewis, R. J.; McCarren, P.; Muncipinto, G.; Norinder, U.; Perry, M. W. D.; Duvall, J. R.; Kihlberg, J. Structural and Conformational Determinants of Macrocycle Cell Permeability. Nat. Chem. Biol. 2016, $12,1065-1074$.

(4) (a) Tsuji, N.; Kennemur, J. L.; Buyck, T.; Lee, S.; Prévost, S.; Kaib, P. S. J.; Bykov, D.; Farès, C.; List, B. Activation of Olefins via Asymmetric Brønsted Acid Catalysis. Science 2018, 359, 1501-1505; (b) Morita, I.; Mori, T.; Mitsuhashi, T.; Hoshino, S.; Taniguchi, Y.; Kikuchi, T.; Nagae, K.; Nasu, N.; Fujita, M.; Ohwada, T.; Abe, I. Exploiting a C-N Bond Forming Cytochrome P450 Monooxygenase for C-S Bond 
Formation. Angew. Chem., Int. Ed. 2020, 59, 3988-3993.

(5) (a) Ball, M. L.; Zhang, B.; Xu, Q.; Paley, D. W.; Ritter, V. C.; Ng, F.; Steigerwald, M. L.; Nuckolls, C. Influence of Molecular Conformation on Electron Transport in Giant, Conjugated Macrocycles. J. Am. Chem. Soc. 2018, 140, 10135-10139.; (b) Grabowski, Z. R.; Rotkiewicz, K.; Rettig, W. Structural Changes Accompanying Intramolecular Electron Transfer: Focus on Twisted Intramolecular Charge-Transfer States and Structures. Chem. Rev. 2003, 103, 3899-4032.

(6) Yamato, T.; Matsumoto, J.; Ide, S.; Tokuhisa, K.; Suehiro, K.; Tashiro, M. Medium-Sized Cyclophanes.

19. Preparation and Conformational Studies of [m.n]Metacyclophanes. J. Org. Chem. 1992, 57, 5243-5246.

(7) (a) Mitchell, R. H. Conformational Changes of 2,11-Dithia[3.3]metacyclophane. A New Look Using VT NMR and Calculation. J. Am. Chem. Soc. 2002, 124, 2352-2357; (b) Witosinska, A.; Musielak, B.; Serda, P.; Owinska, Maria.; Rys, B. Conformation of Eight-Membered Benzoannulated Lactams by Combined NMR and DFT Studies. J. Org. Chem. 2012, 77, 9784-9794.

(8) (a) Ma, H.; Wan, C.; Zewail, A. H. Ultrafast T-Jump in Water: Studies of Conformation and Reaction Dynamics at the Thermal Limit. J. Am. Chem. Soc. 2006, 128, 6338-6340; (b) Hong, C.; Niedzwiedzki, D. M.; Gibson, G. N.; Frank, H. A. Ultrafast Time-Resolved Spectroscopy of Xanthophylls at Low Temperature. J. Phys. Chem. B. 2008, 112, 3558-3567.

(9) Inokuma, Y.; Yoshioka, S.; Ariyoshi, J.; Arai, T.; Hitora, Y.; Takada, K.; Matsunaga, S.; Rissanen, K.; Fujita, M. X-Ray Analysis on the Nanogram to Microgram Scale Using Porous Complexes. Nature 2013, $495,461-466$.

(10) Li, Y.; Tang, S.; Yusov, A.; Rose, J.; Borrfors, A. N.; Hu, C. T.; Ward, M. D. Hydrogen-Bonded Frameworks for Molecular Structure Determination. Nat. Commun. 2019, 10, 4477.

(11) (a) Bounos, G.; Ghosh, S.; Lee, A. K.; Plunkett, K. N.; DuBay, K. H.; Bolinger, J. C.; Zhang, R.; Friesner, R. A.; Nuckolls, C.; Reichman, D. R.; Barbara, P. F. Controlling Chain Conformation in Conjugated Polymers Using Defect Inclusion Strategies. J. Am. Chem. Soc. 2011, 133, 10155-10160; (b) Tang, C.; Tang, Y.; Ye, Y.; Yan, Z.; Chen, Z.; Chen, L.; Zhang, L.; Liu, J.; Shi, J.; Xia, H.; Hong, W. Identifying the Conformational Isomers of Single-Molecule Cyclohexane at Room Temperature. Chem 2020, $6,1-12$.

(12) (a) Li, P.; Xu, S.; Yu, C.; Li, Z.-Y.; Xu, J.; Li, Z.-M.; Zou, L.; Leng, X.; Gao, S.; Liu, Z.; Liu, X.; Zhang, S. De novo Construction of Catenane of Dissymmetric Cages via Space-Discriminative Post-Assembly 
Modification. Angew. Chem., Int. Ed. 2020, 59, 7113-7121; (b) Xu, S.; Li, P.; Li, Z.-Y.; Shi, Z.; Yu, C.; Liu, Z.; Liu, X.; Zhang, S. Catenated Cages Mediated by Enthalpic Raction Intermediates. CCS Chem. 2020, 2, $1838-1850$.

(13) Sun, Z.; Li, P.; Xu, S.; Li, Z.-Y.; Nomura, Y.; Li, Z.-M.; Liu, X.; Zhang, S. Controlled Hierarchical Self-Assembly of Catenated Cages. J. Am. Chem. Soc. 2020, 142, 10833-10840.

(14) (a) Mastalerz M. One-Pot Synthesis of a Shape-Persistent Endo-Functionalised Nano-Sized Adamantoid Compound. Chem. Commun. 2008, 4756-4758; (b) Tozawa, T.; Jones, J. T. A.; Swamy, S. I.; Jiang, S.; Adams, D. J.; Shakespeare, S.; Clowes, R.; Bradshaw, D.; Hasell, T.; Chong, S. Y.; Tang, C.; Thompson, S.; Parker, J.; Trewin, A.; Bacsa, J.; Slawin, A. M. Z.; Steiner, A.; Cooper, A. I. Porous Organic Cages. Nat. Mater. 2009, 8, 973-978; (c) Jin, Y.; Voss, B. A.; Noble, R. D.; Zhang, W. A Shape-Persistent Organic Molecular Cage with High Selectivity for the Adsorption of $\mathrm{CO}_{2}$ over $\mathrm{N}_{2}$. Angew. Chem., Int. Ed. 2010, 49, 6348-6351; (d) Wang, X.; Wang, Y.; Yang, H.; Fang, H.; Chen, R.; Sun, Y.; Zheng, N.; Tan, K.; Lu, X.; Tian, Z.; Cao, X. Assembled Molecular Face-Rotating Polyhedra to Transfer Chirality from Two to Three Dimensions. Nat. Commun. 2016, 7, 12469; (e) Jiao, T.; Chen, L.; Yang, D.; Li, X.; Wu, G.; Zeng, P.; Zhou, A.; Yin, Q.; Pan, Y.; Wu, B.; Hong, X.; Kong, X.; Lynch, V. M.; Sessler, J. L.; Li, H. Trapping White Phosphorus within a Purely Organic Molecular Container Produced by Imine Condensation. Angew. Chem., Int. Ed. 2017, 56, 14545-14550; (f) Ke, X.-S.; Kim, T.; He, Q.; Lynch, V. M.; Kim, D.; Sessler, J. L. Three-Dimensional Fully Conjugated Carbaporphyrin Cage. J. Am. Chem. Soc. 2018, 140, 16455-16459; (g) Duan, H.; Li, Y.; Li, Q.; Wang, P.; Liu, X.; Cheng, L.; Yu, Y.; Cao, L. Host-Guest Recognition and Fluorescence of a Tetraphenylethene-Based Octacationic Cage. Angew. Chem. Int. Ed. 2020, 59, 1010110110; (h) Zhang, G.; Mastalerz, M. Organic Cage Compounds-from Shape-Persistency to Function. Chem. Soc. Rev. 2014, 43, 1934-1947; (i) Hasell, T.; Cooper, A. I. Porous Organic Cages: Soluble, Modular and Molecular Pores. Nat. Rev. Mater. 2016, 1, 1-14.

(15) (a) Rowan, S. J.; Cantrill, S. J.; Cousins, G. R. L.; Sanders, J. K. M.; Stoddart, J. F. Dynamic Covalent Chemistry. Angew. Chem., Int. Ed. 2002, 41, 898-952; (b) Jin, Y.; Yu, C.; Denman, R. J., Zhang, W. Recent Advances in Dynamic Covalent Chemistry. Chem. Soc. Rev. 2013, 42, 6634-6654.

(16) (a) Wang, Y.; Zhang, Y.; Zhou, Z.; Vanderlinden, R. T.; Li, B.; Song, B.; Li, X.; Cui, L.; Li, J.; Jia, X.; Fang, J.; Li, C.; Stang, P. J. A Cyclic bis[2]Catenane Metallacage. Nat. Commun. 2020, 11, 2727; (b) Greenaway, R. L.; Santolini, V.; Szczypinski, F. T.; Bennison, M. J.; Little, M. A.; Marsh, A.; Jelfs, K. E.; Cooper, A. I. Organic Cage Dumbbells. Chem. Eur. J. 2020, 26, 3718-3722. 
(17) (a) Zhang, C.-X.; Wang, Q.; Long, H.; Zhang, W. A. Highly C70-Selective Shape-Persistent Rectangular Prism Constructed Through One-Step Alkyne Metathesis. J. Am. Chem. Soc. 2011, 133, 20995-21001; (b) Mastalerz, M.; Schneider, M. W.; Oppel, I. M.; Presly, O. A Salicylbisimine Cage Compound with High Surface Area and Selective $\mathrm{CO}_{2} / \mathrm{CH}_{4}$ Adsorption. Angew. Chem., Int. Ed. 2011, 50, 1046-1051; (c) Mitra, T.; Jelfs, K. E.; Schmidtmann, M.; Ahmed, A.; Chong, S. Y.; Adams, D. J.; Cooper, A. I. Molecular Shape Sorting Using Molecular Organic Cages. Nat. Chem. 2013, 5, 276-281; (d) Zhang, L.; Jin, Y.; Tao, G.-H.; Gong, Y.; Hu, Y.; He, L.; Zhang, W. Desymmetrized Vertex Design toward a Molecular Cage with Unusual Topology. Angew. Chem. Int. Ed. 2020, 59, 20846-20851.

(18) (a) McCaffrey, R.; Long, H.; Jin, Y.; Sanders, A.; Park, W.; Zhang, W. Template Synthesis of Gold Nanoparticles with an Organic Molecular Cage. J. Am. Chem. Soc. 2014, 136, 1782-1785; (b) Mondal, B.; Acharyya, K.; Howlader, P.; Mukherjee, P. S. Molecular Cage Impregnated Palladium Nanoparticles: Efficient, Additive-Free Heterogeneous Catalysts for Cyanation of Aryl Halides. J. Am. Chem. Soc. 2016, 138, 1709-1716; (c) Uemura, T.; Nakanishi, R.; Mochizuki, S.; Kitagawa, S.; Mizuno, M. Radical Polymerization of Vinyl Monomers in Porous Organic Cages. Angew. Chem. Int. Ed. 2016, 55, 6443-6447; (d) Qiu, L.; McCaffrey, R.; Jin, Y.; Gong, Y.; Hu, Y.; Sun, H.; Parkc, W.; Zhang W. Cage-Templated Synthesis of Highly Stable Palladium Nanoparticles and their Catalytic Activities in Suzuki-Miyaura Coupling. Chem. Sci. 2018, 9, 676-680; (e) Wang, Y.; Sun, Y.; Shi, P.; Sartin, M. M.; Lin, X.; Zhang, P.; Fang, H.; Peng, P.; Tian, Z.; Cao, X. Chaperone-like Chiral Cages for Catalyzing Enantioselective Supramolecular Polymerization. Chem. Sci. 2019, 10, 8076-8082.

(19) (a) Zhang, C.; Patil, R. S.; Liu, C.; Barnes, C. L.; Atwood, J. L. Controlled 2D Assembly of NickelSeamed Hexameric Pyrogallol[4]arene Nanocapsules. J. Am. Chem. Soc. 2017, 139, 2920-2923; (b) ThorpGreenwood, F. L.; Ronson, T. K.; Hardie, M. J. Copper Coordination Polymers from Cavitand Ligands: Hierarchical Spaces from Cage and Capsule Motifs, and Other Topologies. Chem. Sci. 2015, 6, 5779-5792; (c) Bloch, W. M.; Holstein, J. J.; Dittrich, B.; Hiller, W.; Clever, G. H. Hierarchical Assembly of an Interlocked $\mathrm{M}_{8} \mathrm{~L}_{16}$ Container. Angew. Chem. Int. Ed. 2018, 57, 5534-5538. 


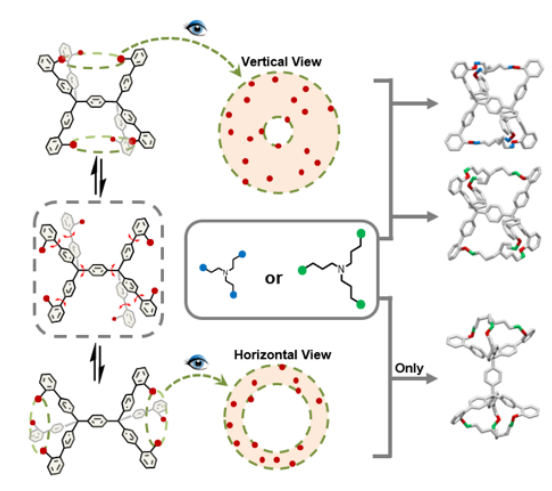

The different-sized clefts of two interconverting conformers were matched and tethered by conformationcapturing molecules with appropriate size. This size-matching strategy not only facilitates the differentiation of conformers that are otherwise undistinguishable with conventional analytical tools, but also led to the discovery of novel cage-like compounds with twin cavities, denoted diphanes. 